\title{
Tissue engineered vascularized periosteal flap enriched with MSC/EPCs for the treatment of large bone defects in rats
}

\author{
CHRISTOPH NAU ${ }^{1}$, DIRK HENRICH ${ }^{1}$, CAROLINE SEEBACH $^{1}$, KATRIN SCHRÖDER $^{2}$, \\ JOHN H. BARKER ${ }^{3}$, INGO MARZI ${ }^{1}$ and JOHANNES FRANK ${ }^{1}$ \\ ${ }^{1}$ Department of Trauma, Hand and Reconstructive Surgery, Johann Wolfgang Goethe-University; \\ ${ }^{2}$ Institute for Cardiovascular Physiology, Goethe-University; ${ }^{3}$ Frankfurt Institute for Regenerative Medicine, \\ Johann Wolfgang Goethe-University, Frankfurt/Main, Germany
}

Received July 10, 2016; Accepted February 2, 2017

DOI: 10.3892/ijmm.2017.2901

\begin{abstract}
Vascularized periosteal flaps are used for complex cases if the reconstruction of large bone defects is necessary in modern trauma and orthopedic surgery. In this study, we combined this surgical procedure with $\beta$-TCP scaffold and mesenchymal stem cells (MSCs) + endothelial progenitor cells (EPCs) as a tissue engineering approach to obtain optimum conditions for bone healing in rats. A critical size femoral defect was created in 80 rats allocated into 4 groups. Defects were treated according to the following protocol: i) vascularized periosteal flap alone; ii) vascularized periosteal flap $+\beta$-TCP scaffold; iii) vascularized periosteal flap $+\beta$-TCP scaffold + ligated vascular pedicle; and iv) vascularized periosteal flap $+\beta$-TCP scaffold + MSCs/EPCs. After 8 weeks, femur bones were extracted and analyzed for new bone formation, vascularization, proliferation and inflammatory processes and strength. Bone mineral density (BMD) and biomechanical stability at week 8 were highest in group 4 $($ flap $+\beta$-TCP scaffold + MSCs/EPCs) compared to all the other groups. Stability was significantly higher in group 4 (flap $+\beta$-TCP scaffold + MSCs/EPCs) in comparison to group 3 (ligated flap $+\beta$-TCP scaffold). BMD was found to be significantly lower in group 3 (ligated flap $+\beta$-TCP scaffold) compared to group 1 (flap) and group 4 (flap $+\beta$-TCP scaffold + MSCs/EPCs). The highest density of blood vessels was observed in group 4 (flap $+\beta$-TCP + MSCs/EPCs) and the values were significantly increased in comparison to group 3 (ligated flap), but not to group 1 (flap) and group 2 (flap $+\beta$-TCP). The
\end{abstract}

Correspondence to: Dr Christoph Nau, Department of Trauma, Hand and Reconstructive Surgery, Hospital of the Johann Wolfgang Goethe-University, Theodor-Stern-Kai 7, D-60590 Frankfurt/Main, Germany

E-mail: christoph.nau@kgu.de

Key words: tissue engineering, vascularized periosteal flap, mesenchymal stem cells, endothelial progenitor cells, large bone defects, cell transplantation highest amounts of proliferating cells were observed in group 4 (flap $+\beta$-TCP scaffold + MSC/EPCs). The percentage of proliferating cells was significantly higher in group 4 (flap $+\beta$-TCP scaffold + MSCs/EPCs) in comparison to all the other groups after 8 weeks. Our data thus indicate that critical size defect healing could be improved if MSCs/EPCs are added to $\beta$-TCP scaffold in combination with a periosteal flap. Even after 8 weeks, the amount of proliferating cells was increased. The flap blood supply is essential for bone healing and the reduction of inflammatory processes.

\section{Introduction}

When the intrinsic ability of bone to regenerate is overpowered by large segmental defects, the patients who suffer with these defects and have to manage long term operations and treatments, physicians who have to perform complex surgical procedures and the health care system which has to bear high costs for these procedures are faced with major challenges.

While many approaches have been developed to treat these difficult large bone defects, none of these have proven to be fully satisfactory. The most commonly used treatments employ autologous bone, either non-vascularized $(1,2)$ or vascularized. Several biodegradable bone graft substitutes have been developed and are commercially available (3-5). Other treatments include, the Ilizarov technique (6) and vascularized periosteal flaps $(7,8)$.

Limitations associated with current treatments have led to the search for alternative treatments using cell-based tissue engineering protocols $(9,10)$. While still largely experimental, these methods have generated great interest in research, industry and clinical settings with particular emphasis on treatments for bone defects $(11,12)$. Generally these approaches consist of combining biodegradable scaffolds with different combinations of bone- and vessel-forming growth factors and cells.

Biodegradable scaffolds or 'bone graft substitutes' as their name implies, have been developed to replace autologous bone grafts with their associated limitations and complications like, limited donor bone availability and donor site morbidity. These substitutes have been specifically engineered to integrate with bone tissue while providing structural 3D support (13). While 
they have been successful in reducing the need for autologous bone, their ability to duplicate bone osteogenesis and vessel angiogenesis capacity of autologous bone grafts has yet to be conclusively demonstrated (14).

We, as well as others have explored the use of cell-based approaches, which involve seeding scaffolds with different combinations of bone- and vessel-forming cells and growth factors $(12,15-18)$. The advantages of this approach are obvious, as it delivers bone-forming cells and growth factors directly into the bone defect where they are needed.

Mesenchymal stem cells (MSCs) have been extensively studied due to their known potential to differentiate into chondrogenic and/or osteogenic cells, the main cellular mediators of bone formation. Using this approach, seeding scaffolds with MSCs into bone defects in animal models, we as well as others have demonstrated mostly positive results (18-20). Despite these encouraging initial results, using this approach, the bone-forming capacity observed when autologous bone grafts are used has not yet been achieved. The reasons for this may be the limited viability of the transplanted MSCs, the lack of osteoinductive stimuli and a lack of angiogenesis. In order to address these issues, investigators have used genetically modified MSCs (21), using different proteins including, bone morphogenic protein (BMP) $(12,15)$, vascular endothelial growth factor (VEGF) (22) and fibroblast growth factor (FGF) (23). Thus far, optimal results have been obtained when using a combination of osteogenic and angiogenic proteins $(24,25)$.

Endothelial progenitor cells (EPCs) are of hematopoietic origin, and are known to participate in angiogenesis (26). It has been shown that EPCs home to ischemic tissue, stimulate blood flow recovery in ischemic tissues, and are increased in numbers in the blood following trauma, all qualities that make EPC logical candidates for treating large bone defects $(27,28)$. Keeping this in mind, we combined MSCs as a source for osteogenic activity and EPCs due to their pro-angiogenic activity. In a previous study, we treated large rat femur bone defects with scaffolds seeded with a combination of MSCs and EPCs and observed a much higher bone healing response in animals that received both MSCs and EPCs (18).

Although these tissue-engineering approaches that combine cells, osteoinductive proteins and osteoconductive scaffolds have been encouraging, they have not yet achieved widespread clinical acceptance. This may be due to the logistics of applying these new techniques in the clinical setting, but also as they have not conclusively demonstrated clinical superiority over autologous bone grafts $(18,29,30)$.

Vascularized periosteal flaps, introduced by Doi and Sakai in the early 1990s, consist of transferring a thin 'flap' of periosteum with its intact blood supply to cover bone defects (7). The rich blood supply of these flaps, together with their thin and pliable structure makes them very versatile and has enabled surgeons to successfully use them to reconstruct bone defects in a variety of different anatomical locations. The periosteum is a rich source of blood supply and boneforming cells. Its outer 'fibrous' layer contains a nerve and blood supply, while its' inner 'cellular' layer contains different kinds of stem cells (31-33). This inner cellular layer becomes progressively thinner with age. In adults the periosteum becomes so thin that it cannot be distinguished from the overlying fibrous layer $(34,35)$.
We evaluated the effects of a periosteal flap together with a tissue engineering approach in a former study (36), and in the present study, we sought to combine these new cell-based MSC/EPC therapy approaches with the well-established and clinically proven method of a vascularized periosteal flap. This combination which has not been performed in the past by any other research group, at least to the best of our knowledge, would bring together the recently demonstrated benefits of tissue engineering approaches with the well-established clinical success of periosteal flaps. The healing of critical size bone defects could be facilitated by this method, since bone grafts with their donor site morbidity would no longer be necessary and patients would not have to cope with long-term external fixation, such as that associated with the Ilizarov technique.

\section{Materials and methods}

Animal care. All experiments were performed in accordance with regulations established and approved (project no. F3/21; Regierungspräsidium, Darmstadt, Germany) by our Institutional Animal Care and Oversight Committee according to German law. Ten-week-old male Sprague-Dawley (SD) rats (Harlan Cytotest Cell Research GmbH, Rossdorf, Germany) weighing 350-400 g were used. Animals were caged individually in temperature $\left(21^{\circ} \mathrm{C}\right)$, light $(12 \mathrm{~h}$ light, $12 \mathrm{~h}$ dark), and air flow-controlled rooms. They received standard rodent chow and water containing tramadol pain killer ad libitum post-operatively. Rats were monitored daily for complications or abnormal behavior during the post-operative period.

Group setup. A total of 80 rats (SD; Harlan Cytotest Cell Research $\mathrm{GmbH}$ ) were allocated into 4 groups, consisting of 20 animals/group. Critical size defects were created on their femur bones and were treated as follows: group 1, vascularized periosteal flap alone; group 2, vascularized periosteal flap $+\beta$-TCP scaffold; group 3 , periosteal flap (with ligated vascular pedicle) $+\beta$-TCP scaffold; group 4 , vascularized periosteal flap $+\beta$-TCP scaffold + MSCs/EPCs.

After 8 weeks, the rat femurs were harvested and all bones were examined using radiological and immunohistochemistry methods. Eight rats were used for histological analysis and 6 were used for micro-CT and biomechanical testing. Biomechanical three-point bending tests were performed in bones, which had been previously used for radiological examination.

\section{Animal model}

Critical size femur bone defect. Under general anesthesia [ketamine chlorhydrate $(100 \mathrm{mg} / \mathrm{kg})$ and xylazine hydrochloride $(10 \mathrm{mg} / \mathrm{kg})]$ administered intraperitoneally, the right legs of the rats were shaved, cleaned and disinfected with antiseptic fluid. A medial longitudinal incision was made through the skin and fascia over the femur and the underlying vastus medialis and biceps muscles were separated bluntly. The ventral aspect of the femur bone was exposed. In order to provide stability to the bone after creation of the defect $1.5 \mathrm{~mm}$ 5-screw stainless-steel plates Compact Hand (Synthes GmbH, Umkirch, Germany) were secured to 
the femur bone using $1.5 \mathrm{~mm}$ cortical screws. A 7-mm-long bone defect was then created using a drill in the mid-shaft of the femur between the second and the third screw. Special care was taken to ensure that no bone fragments were left underneath the plate along the 7-mm defect, as previously described (18).

The periosteum overlaying the medial femoral condyle and its blood supply (descending genicular artery and vein) were identified, exposed and the medial condyle periosteal flap was elevated on its vascular pedicle. The vascular pedicle was carefully dissected along a trajectory of $15 \mathrm{~mm}$ and the flap was then rotated into the defect and fixed with sutures (5-0 Vicryl; Ethicon, Norderstedt, Germany) to the medial side of the plate to bridge the defect. The lateral side of the defect was left free. After the femur bone defects in the different groups had received their respective treatments, the fascia was re-approximated with interrupted 5-0 Vicryl sutures (Ethicon), and the skin sewed up with intracutaneous sutures (4-0 Prolene; Ethicon).

Scaffold preparation and implantation into defect. A commercially available bone graft substitute (chronOS $\beta$-TCP, size $0.7-1.4 \mathrm{~mm}$, porosity $60 \%$ and pore size $100-500 \mu \mathrm{m}$; Synthes $\mathrm{GmbH}$ ) was incubated for $30 \mathrm{~min}$ in a fibronectin solution (10 $\mu \mathrm{g} / \mathrm{ml}$; Sigma, Deisenhofen, Germany) in phosphate-buffered saline (PBS) without $\mathrm{Mg}^{2+}$ and $\mathrm{Ca}^{2+}\left(\mathrm{PBS}^{-/-}\right)$. The supernatant was then removed after $30 \mathrm{~min}$ and replaced by $\mathrm{PBS}^{-/-}$only. The granules were immediately placed, as a dense single layer, in a 24 -well plate (Nunc, Wiesbaden, Germany) using sterile forceps. Fibronectin is a commonly used and accepted substrate, shown to support EPC differentiation and adherence (37). In a previous study we found that fibronectin coating enhanced EPC adherence to $\beta$-TCP (17). This preparation was then used to fill the femur bone defect prior to implanting the periosteal flap.

EPC and MSC harvest, isolation and characterization. Rat early EPCs were isolated from the spleens of syngeneic male SD rats according to previously described procedures $(38,39)$. The advantage in doing so is that the absolute number of harvested cells is much higher if spleen is used in comparison to bone. The spleen was therefore cut into small sections (approximately $3 \mathrm{~mm}$ ) and gently mashed using syringe plungers. The cell suspension was then filtered through a $100 \mathrm{~mm}$ mesh, washed once with PBS and subjected to Ficoll density gradient centrifugation $(30 \mathrm{~min}, 900 \mathrm{x} \mathrm{g}$ with Ficoll $1.077 \mathrm{~g} / \mathrm{ml}$; Biochrom, Berlin, Germany). Recovered mononuclear cells were washed twice with cold $\mathrm{PBS}^{\text {w/o }}$ (10 min, $900 \times \mathrm{g}$ ), and then each $4 \times 10^{6}$ cells were cultivated on a fibronectin-coated (10 $\mu \mathrm{g} / \mathrm{ml}$; Sigma) 24-well culture dish in $1 \mathrm{ml}$ of endothelial basal medium supplemented with endothelial growth medium (both from Cambrex, Verviers, Belgium) with singlequots at $37^{\circ} \mathrm{C}, 5 \% \mathrm{CO}_{2}$. After $48 \mathrm{~h}$, non-adherent and weakly-adherent cells were removed, the medium was changed, and the cells were cultivated for an additional $72 \mathrm{~h}$. Moreover, a parallel preparation was performed to evaluate the percentage of endothelial-like differentiated cells. EPCs were identified using a previously described method $(40,41)$. Briefly, the cells were incubated with $2.4 \mu \mathrm{g} / \mathrm{ml}$ DiLDL (Cell Systems, St. Katharinen, Germany) in EBM supplemented with $20 \%$ fetal calf serum (FCS) for $1 \mathrm{~h}$. Cells were then fixed with $2 \%$ paraformaldehyde for $10 \mathrm{~min}$ and, after washing with
$\mathrm{PBS}^{+/+}$, were incubated with FITC labeled Ulex europaeus agglutinin-1 (10 $\mu \mathrm{g} / \mathrm{ml})$ (lectin; Sigma) for $1 \mathrm{~h}$. Cells presenting double-positive fluorescence (1,1'-dioctadecyl-3,3,3',3'-tetramethylindocarbocyanine-labeled acetylated low-density lipoprotein, lectin) were considered to be EPCs. For these experiments, the cells were detached by accutase treatment (10 min) (PAA Laboratories, Linz, Austria), washed once with MesenCult + Supplements (Cell Systems), and subsequently adjusted to a density of $2.5 \times 10^{5}$ cells in $100 \mu \mathrm{l}$.

MSCs were obtained from donor rat femurs. More precisely, they were isolated from femurs obtained from syngeneic donor rats by flushing the bone marrow with PBS. The bone marrow was then re-suspended in PBS and washed once by centrifugation $(8 \mathrm{~min}, 300 \mathrm{x} \mathrm{g}$ ). The cells obtained from each femur were subsequently plated into individual $75 \mathrm{~cm}^{2}$ culture flasks using DMEM supplemented with 10\% FCS (Gibco, Darmstadt, Germany) and expanded over three passages. Subsequently, the cells were detached by accutase (PAA Laboratories) treatment, washed, and re-suspended in a medium consisting of $90 \%$ FCS and $10 \%$ dimethyl sulfoxide (Sigma). Aliquots were stored in liquid nitrogen until use.

For each experiment, a portion of the cryoconserved cells was thawed and expanded over two additional passages. MSCs of the sixth culture passage (P6) were then used for the experiments. After accutase treatment $(10 \mathrm{~min})$, cells were washed (10 min, $300 \mathrm{x} \mathrm{g}$ ) and re-suspended in PBS. Hereafter, the cell suspension was divided, and one part was adjusted to a density of $2.5 \times 10^{5}$ cells in $100 \mu \mathrm{l}$ to be used in the experiments. The other portion was used to assess MSC osteogenic differentiation potential by incubating the cells in osteogenic differentiation medium containing dexamethason $\left(1 \times 10^{-6} \mathrm{M}\right)$, ascorbic acid $(50 \mu \mathrm{g} / \mathrm{ml})$, and $\beta$-glycerol phosphate $\left(1 \times 10^{-1} \mathrm{M}\right)$ for 3 weeks. The medium was exchanged twice a week. Osteogenic substances were purchased from Stem Cell Technologies (Grenoble, France). Extracellular calcium deposition was evaluated using von Kossa staining (18).

EPC/MSC seeding on scaffolds. Granules were densely placed as bilayer into individual wells of 48-well plates. Hereafter, the granules were loaded with either $5 \times 10^{5}$ cells of a mixture composed of 50\% MSCs and 50\% EPCs or with $5 \times 10^{5}$ MSCs or $5 \times 10^{5}$ EPCs alone (17). The cells were dripped $(200 \mu \mathrm{l})$ over the bone graft layer and incubated for $10 \mathrm{~min}$ at $37^{\circ} \mathrm{C}$. The medium containing the non-adhering cells was then removed, rinsed once again over the granules, and incubated at $37^{\circ} \mathrm{C}$ for a further $10 \mathrm{~min}$. This procedure was repeated 3 times. Subsequently, the granules loaded with cells were subjected to the animal facility. Granules were constantly kept at $37^{\circ} \mathrm{C}$ and were implanted into the bone defects within $2 \mathrm{~h}$ after seeding. To confirm adherence of EPCs and MSCs on biomaterials we used a parallel setup. DiLDL pre-stained EPCs and MSCs were seeded on the granules as described. Subsequently, the cells were fixed with $2 \%$ paraformaldehyde in $\mathrm{PBS}^{+/+}$for $20 \mathrm{~min}$ and washed gently with $2 \mathrm{X} 200 \mu \mathrm{l}$ PBS per well and followed by further incubation with $1 \mu$ l DAPI [2-(4-amidinophenyl)-6-indolecarbamidine dihydrochloride; Sigma] with a final concentration of $1 \mu \mathrm{g} / \mathrm{ml}$ for $10 \mathrm{~min}$ at room temperature. After the staining, the granules were washed three times with $\mathrm{PBS}^{-/}$and transferred to a new well in order to prevent false-positive results caused by the adherent cells at 
the bottom of the cultivation well. Finally, the granules were analyzed by fluorescence microscopy (Axio Observer; Carl Zeiss, Inc., Göttingen, Germany) and photographed. EPCs appear orange and MSCs appear blue (Fig. 1).

Euthanasia. The animals were euthanized with an overdose of pentobarbital ( $150 \mathrm{mg} / \mathrm{kg}$ intraperitoneally) after 8 weeks. The animal femurs were dissected free and all bones were examined macro- and microscopically for signs of infection or tumors. Bones were then frozen and stored at $-80^{\circ} \mathrm{C}$ until preparation for immunhistological examinations. Thereafter, the bones were fixed in Zinc-Formal-Fixx (4\%; Thermo Electron, Pittsburgh, PA) $>20 \mathrm{~h}$ and then subjected to decalcification in a solution containing $0.25 \mathrm{M}$ Trizma base (Sigma) (17) for 14 days.

Measurements. Various measurements were performed 8 weeks after surgery as follows:

Bone maturation, vascularization and inflammation in the defect zone. Samples taken from the bone defect zone were decalcified, fixed in $4 \%$ formaldehyde and embedded in paraffin. Sections were stained with hematoxylin and eosin or incubated with antibodies directed against osteocalcin (bone maturation, dilution 1:100, incubation time $1 \mathrm{~h}, 4^{\circ} \mathrm{C}$; Abcam, Cambridge, UK), CD31 (blood vessels, dilution 1:50, incubation overnight, $4^{\circ} \mathrm{C}$; Abcam), Ki-67 (proliferation, M7248, dilution 1:50, incubation time $1 \mathrm{~h}, 4^{\circ} \mathrm{C}$; Dako, Glostrup, Denmark) and HLA-DR (inflammation, ab23990, dilution 1:100, incubation time $1 \mathrm{~h}, 4^{\circ} \mathrm{C}$; Abcam). Polyclonal HRP-coupled secondary antibodies were applied and the sections were incubated with 3-amino-9-ethyl-carbazole (AEC).

Osteocalcin staining in the defect zone was evaluated purely descriptive using stitched high resolution images of the whole defect area using a Keyence Biorevo BZ-9000 microscope and the software BZ II analyzer (Keyence Deutschland GmbH, Neu-Isenburg, Germany).

For thedetermination of blood vessel density, the number of blood vessels (CD31) with a lumen was counted in 5 non-overlapping images of the defect area at a magnification of $x 100$ in combination with a computer-supported imaging picture analysis system (Axiovision 4.7; Carl Zeiss, Inc.). Values were converted to blood vessel $/ \mu \mathrm{m}$ and normalized to the percentage of tissue in the analysed image frame.

Histological Ki-67 and HLA-DR staining was evaluated in the medial and lateral aspect of the defect zone. The percentage of Ki-67-positive cells was determined in a standardized area, that was normalized to the area covered by tissue. The values were ascertained to 5 categories (0, no $\mathrm{Ki}-67$-positive cells; 1 , up to $25 \%$; 2, 25-50\%; 3, 50-75\%; 4, 75-100\% Ki-67-positive cells). The percentage HLA-DR-positive area was determined using ImageJ software (https://imagej.nih.gov/ij/) and normalized to the area covered by tissue in the defect zone. All histological slides were analyzed in a random order by an independent observer blinded to the group setup $(17,18)$.

Peripheral quantitative computed tomography ( $p Q C T)$ of bone defects. To assess bone density and micro architecture at the defect site pQCT and micro-CT (SkyScan 1176; Bruker Corp., Billerica, MA, USA) were performed on 6 of the 20 femurs harvested at 8 weeks. For imaging, femurs were oriented along their long axis orthogonally to the axis of the X-ray beam (90 kV X-ray source, fully distortion corrected 11-megapixel X-ray camera). Two-dimensional CT images were scanned and reconstructed using a standard convolution-back-projection procedure. The isotropic voxel size was $18 \mu \mathrm{m}$. The analyzed volume of interest was placed at the center of the bone defect with $0.7 \mathrm{~mm}$ thickness for all samples (18). $\beta$-TCP signals were not subtracted from bone mineral density (BMD) values.

Biomechanical testing. In bones (6 in total), that had already been used for pQCT, biomechanical properties, at the defect site were measured by a destructive three-point bending procedure using a material testing machine (zwickiLine Z5.0; Zwick-Roell, Ulm, Germany). The 'bending until failure' method was performed by lowering a bar onto the femur, using a constant deflection speed of $0.1 \mathrm{~mm} / \mathrm{sec}$, and recording the load and deflection continuously. The ultimate load was then calculated using testXpert II software (Zwick-Roell) (18).

Statistical analysis. In this study, differences between the groups were compared using the non-parametric KruskalWallis test followed by multiple Conover-Iman comparison with Bonferroni-Holm correction. The software BIAS 10.11 (Epsilon, Darmstadt, Germany) was used for group size calculation. A value of $\mathrm{P}<0.05$ was considered to indicate a statistically significant difference.

\section{Results}

No deaths occurred during surgery or during the immediately following post-operative period. Furthermore, the animals exhibited no abnormal behavior during daily monitoring.

Biomechanical results and BMD. The median BMD values and biomechanical stability at week 8 were highest in group 4 $($ flap $+\beta$-TCP scaffold + MSCs/EPCs) compared to all the other groups. However, as regards group 4 , this was only significant compared to group 3 (ligated flap $+\beta$-TCP scaffold). The median BMD values and stability in group 4 (flap $+\beta$-TCP scaffold + MSCs/EPCs) were higher when compared to group 2 (flap $+\beta$-TCP scaffold), although not significantly. Stability was significantly higher in group 4 (flap $+\beta$-TCP scaffold + MSCs/EPCs) in comparison to group 3 (ligated flap $+\beta$-TCP scaffold), in which no stability was observed at all. In addition, group 1 (flap) and group 2 (flap $+\beta-$ TCP scaffold) presented higher biomechanical stability compared to group 3 (ligated flap $+\beta$-TCP scaffold). BMD was found to be significantly lower in group 3 (ligated flap $+\beta$-TCP scaffold) compared to group 1 (flap) and group 4 (flap $+\beta-\mathrm{TCP}$ scaffold + MSCs/EPCs), and lower (although not significantly) compared to group 2 (flap $+\beta$-TCP scaffold) (Fig. 2)

Gross histological analysis. A thick bony flap was found in group 1 (flap alone) and bone began to grow into the defect zone originating from the flap. However, no healing was observed after 8 weeks. A calcified flap was also observed in group 2 $($ flap $+\beta$-TCP) similar to group 1. Furthermore, signs of bone formation could be found in the defect zone. However, in group 3 (ligated flap $+\beta$-TCP), the flap appeared completely necrotic and no signs indicating bone healing were observed. Fibrous, loose tissue formed in the defect instead, and signs of infection were present in two bones. In group 4 (flap $+\beta-\mathrm{TCP}+\mathrm{MSC} / \mathrm{EPCs}) \mathrm{a}$ 

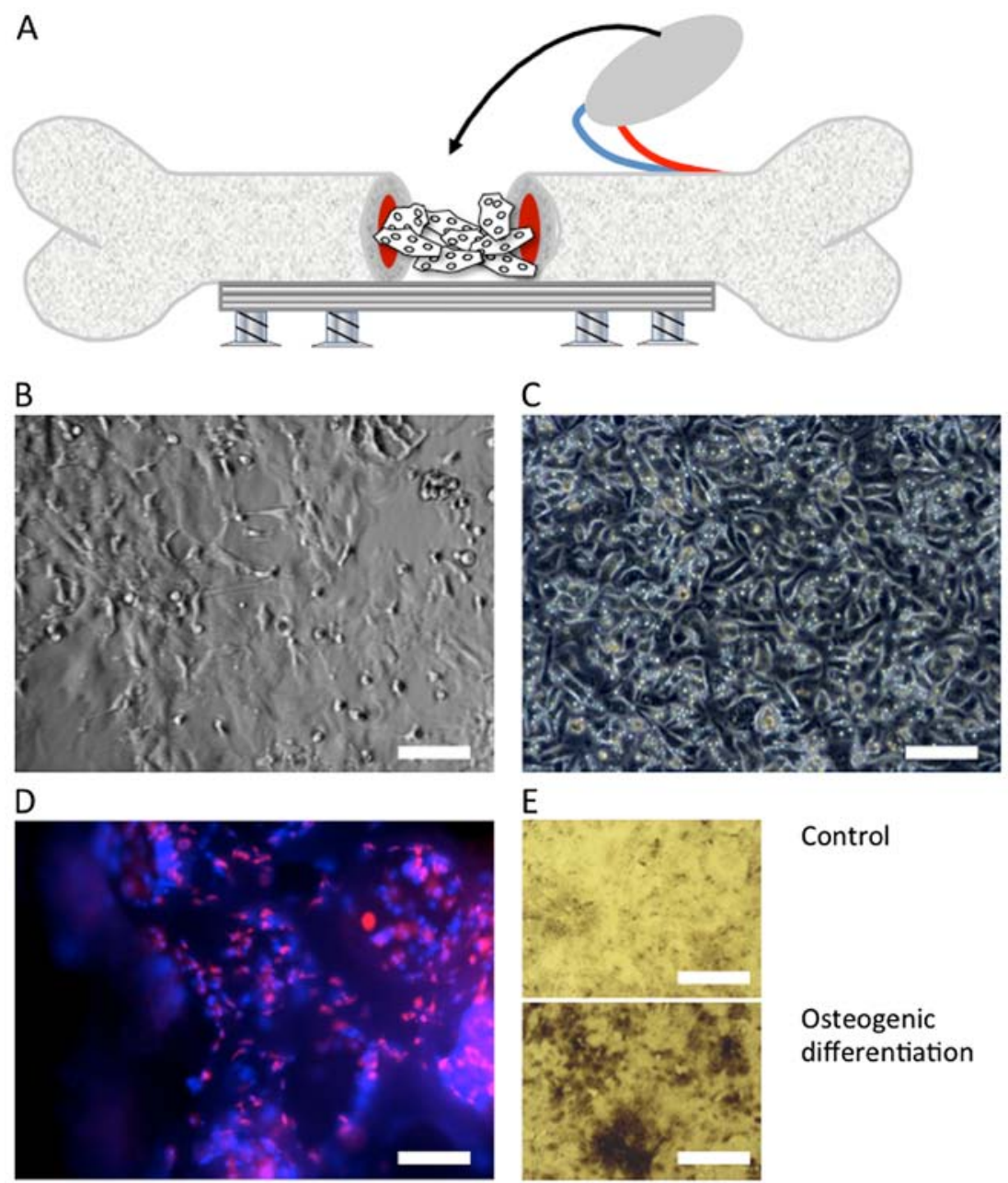

Control

Osteogenic

differentiation

Figure 1. (A) Principal of vascularized periosteal flap transfer into the stabilized large bone defect. A vascularized periosteal flap prepared and pivoted into the bone defect is shown. The filling of the bone defect depends on the respective group assignment. Phase contrast images of (B) MSCs and (C) EPCs isolated from donor rats. MSCs were harvested from femoral bone marrow and EPCs were harvested from rat spleen from donor SD rats as described in the 'Materials and methods'. MSCs of the sixth culture passage (P6) and EPCs of the first passage were used for the experiments. MSCs mainly demonstrated a spindle-shaped morphology, whereas EPCs grew into a typical cobblestone-like pattern. (D) Fluorescence microscopic composite image of MSCs and EPCs seeded on a $\beta$-TCP scaffold is presented. EPCs were pre-stained with DiLDL and appear orange, MSCs were stained with DAPI and appear blue. (E) Calcium deposition of osteogenic differentiated MSCs as measured by von Kossa staining. Calcified areas are shown in black. White bar indicates (B and C) $100 \mu \mathrm{m}$, (D) $200 \mu \mathrm{m}$, (E) $400 \mu \mathrm{m}$. MSCs, mesenchymal stem cells; EPCs, endothelial progenitor cells.
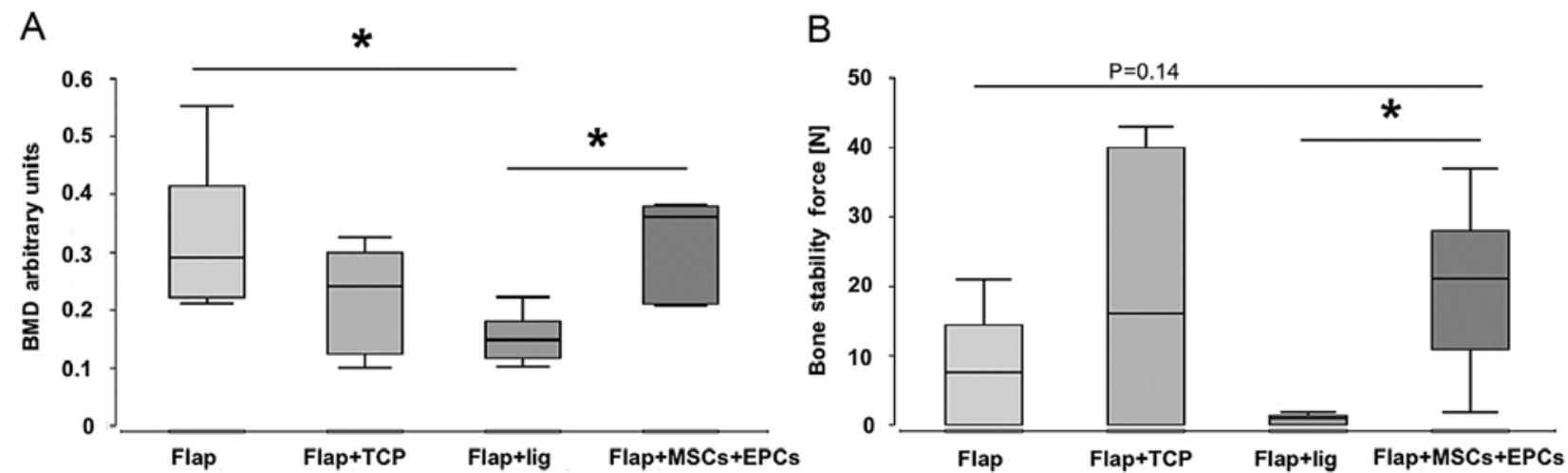

Figure 2. (A) BMD and (B) biomechanical stability were evaluated in the defect area 8 weeks after surgery by $\mu$ CT. Values of BMD were significantly decreased in bone defects treated with the ligated periosteal flap in comparison to animals receiving the vascularized periosteal flap respectively periosteal flap supplemented with $\beta$-TCP combined with MSC and EPC. (B) Ultimate load of the defect zone was determined by three-point bending test. ${ }^{*} \mathrm{P}<0.05$. BMD, bone mineral density; MSCs, mesenchymal stem cells; EPCs, endothelial progenitor cells.

thick calcified flap was detected and bone formation was found throughout the entire defect zone (Fig. 3A-D).
Bone formation and healing. Bone formation was observed in close proximity to the periosteal flap in all cases at 8 weeks, 

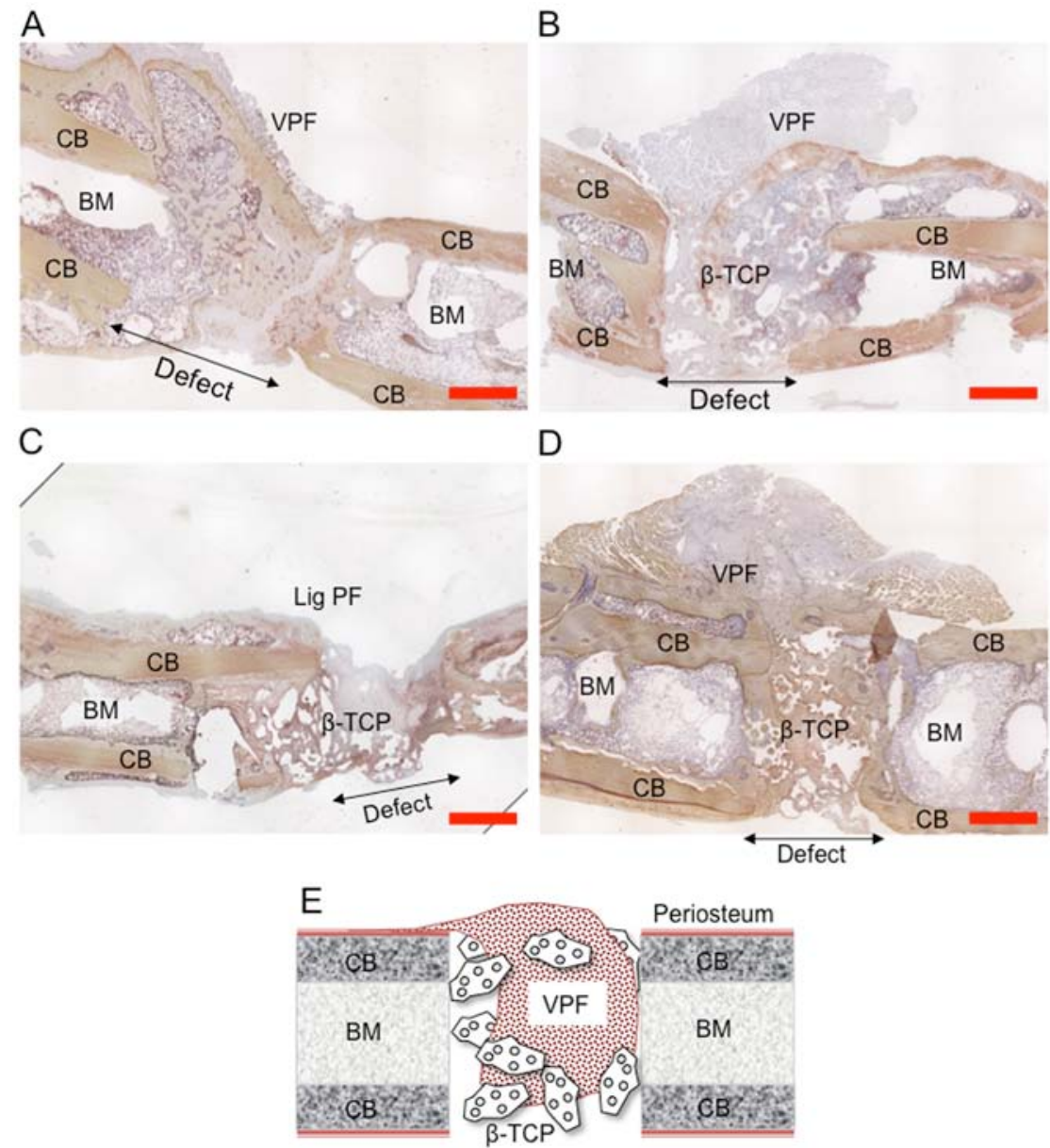

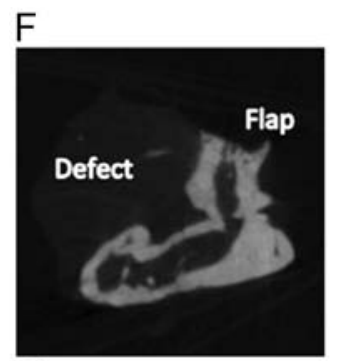

Flap

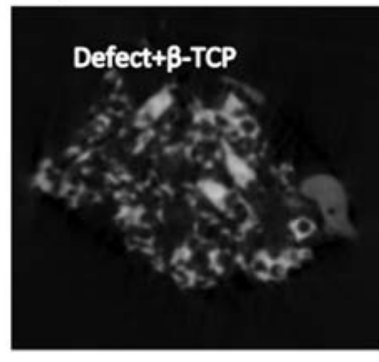

Ligated Flap $+\beta-\mathrm{TCP}$

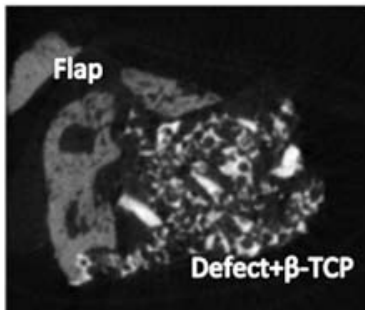

Flap $+\beta-$ TCP

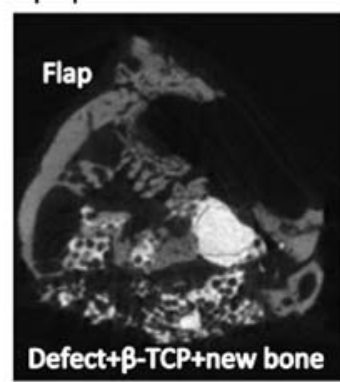

Flap $+\beta-T C P+M S C s / E P C s$ except for defects treated with flaps with ligated vascular pedicles. In groups 1, 2 and 4, with varying degrees, new bone formation could be seen protruding from the flap, as well as in the defect zone. Histological analysis of osteocalcin immunostained slides confirmed this observation, demonstrating a close correlation between the periosteal flap and cortical bone formation in all cases at 8 weeks. All defects treated
Figure 3. Representative osteocalcin-stained histological slides of the defect zone 8 weeks after surgery. Histological slides of the femur defect of (A) group 1 (periosteal flap), (B) group 2 (periosteal flap $+\beta$-TCP), (C) group 3 (ligated periosteal flap $+\beta$-TCP and (D) group 4 (periosteal flap $+\beta$-TCP + MSCs + EPCs) are shown. Brownish color indicates osteocalcin-positive areas. The vascularized periosteal flap demonstrates a high degree of osteogenic differentiation. Pronounced osteogenic differentiation in the defect zone was seen in (A) group 1 and (B) group 4, whereas (C) minimal bone formation and resorption of the periosteal flap was seen, if the periosteal flap was ligated. Defect size is $5 \mathrm{~mm}$. (E) Schematic overview over the defect zone is shown. Original defect size is $5-6 \mathrm{~mm}$. Red bar indicates a distance of $2 \mathrm{~mm}$. (F) Representative $\mu \mathrm{CT}$ images taken from the defect zone 8 weeks after surgery. BM, bone marrow; $\mathrm{CB}$, cortical bone; VPF, vascularized periosteal flap; LigPF, ligated periosteal flap; MSCs, mesenchymal stem cells; EPCs, endothelial progenitor cells. with vascularized flaps revealed thick bony tissue formation (groups 1, 2, 4). Bone density, thickness and integration into the defect zone were visibly greater in group 4 (flap $+\beta$-TCP scaffold + MSCs/EPCs). By contrast, in animals in which the vascular pedicle of the periosteal flap was ligated (group 3), minimal bone formation was observed at the defect, and the periosteal flap was found to be completely reabsorbed. Finally, 


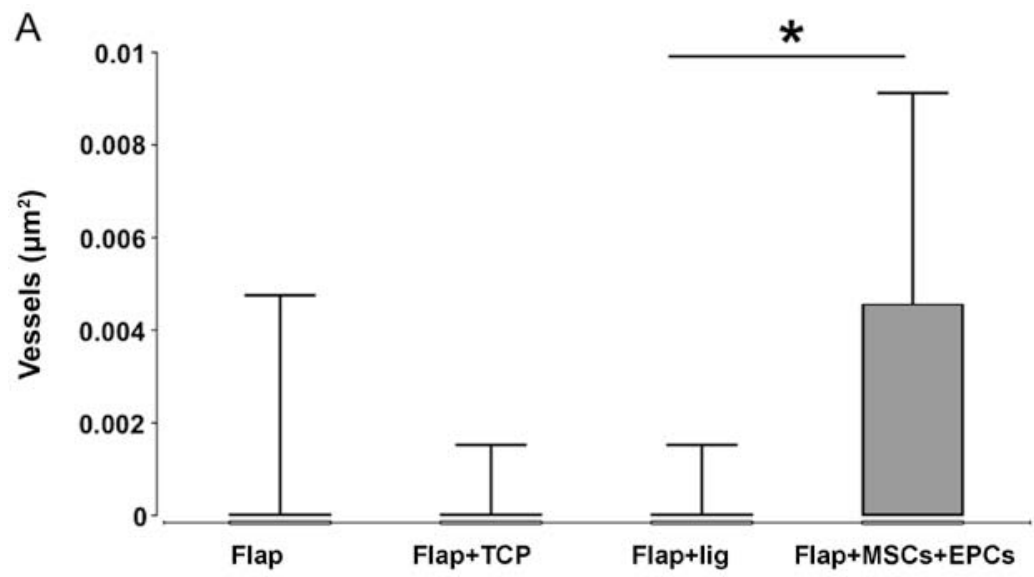

B

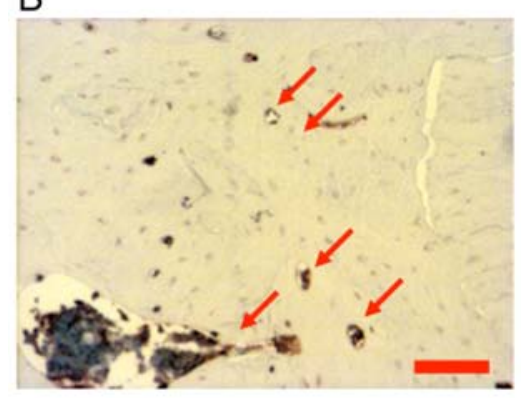

D

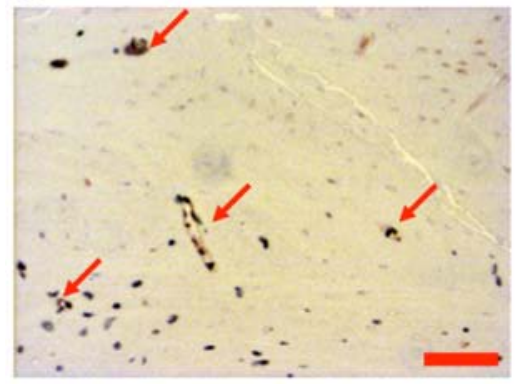

C

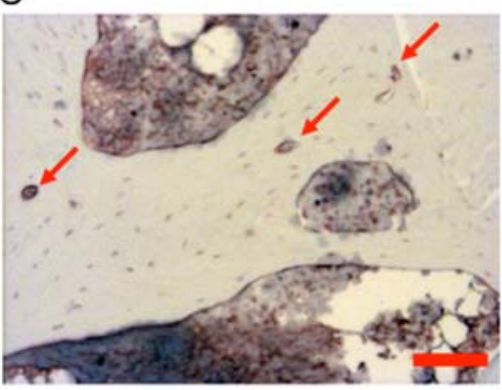

E

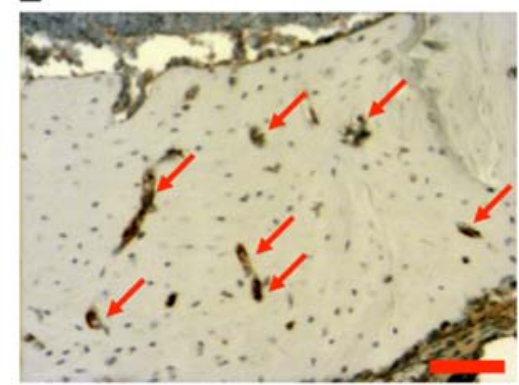

Figure 4. Vascularization in the defect area 8 weeks after surgery as determined by immunohistological evaluation of CD31-stained histological slides. (A) Values are presented as blood vessel density per $\mu \mathrm{m}^{2}$. Representative histological slides of the medial side of the femur defect of (B) group 1 (periosteal flap), (C) group 2 (periosteal flap $+\beta$-TCP), (D) group 3 (ligated periosteal flap $+\beta$-TCP) and (E) group 4 (periosteal flap $+\beta$-TCP + MSCs + EPCs) 8 weeks after surgery are shown. Blood vessels are indicated by arrows. Red bar represents $100 \mu \mathrm{m}$. ${ }^{*} \mathrm{P}<0.05$. MSCs, mesenchymal stem cells; EPCs, endothelial progenitor cells.

$\beta$-TCP resorption appeared to be more advanced in these animals (Fig. 3A-E).

The results observed in histological analysis were confirmed by $\mu \mathrm{CT}$-analysis. In groups 1,2 and 4 , the thick bony flap and increased healing were observed in the defect, while in group 3 (ligated vascular flap), only loose non-calcified tissue was observed (Fig. 3F).

Vascularization. A highest density of blood vessels was observed in group 4 (flap $+\beta$-TCP + MSCs/EPCs) and the values were significantly increased in comparison to group 3 (ligated flap), but not to group 1 (flap) and group 2 $($ flap $+\beta-T C P)($ Fig. 4A-E).

Proliferation and inflammation. Proliferating cells were detected by Ki-67 immunostaining. The greaterst amounts of proliferating cells were observed in group 4 (flap $+\beta$-TCP scaffold + MSCs/EPCs). The percentage of proliferating cells was significantly higher in group 4 (flap $+\beta$-TCP scaffold + MSCs/EPCs) in comparison to all the other groups after 8 weeks at the medial and the lateral defect side (Fig. 5A-F).

HLA-DR was used to reveal signs of infection. The amount of inflammatory cells was highest in group 3 (ligated vascular pedicle) in comparison to all the other groups and significantly higher compared to group 1 (flap) and in trend towards group 2 (flap $+\beta$-TCP scaffold) $(\mathrm{P}=0.056)$ at the lateral side of the defect. At the medial side where the flap is located, the numbers of inflammatory cells were significantly increased in group 3 (ligated vascular pedicle) compared to group 1 (flap) and group 2 (flap $+\beta$-TCP scaffold). A detailed view revealed that HLA-DR-positive cells were not evenly distributed in the tissue of the defect zone, but had accumulated around the $\beta$-TCP granules (groups 2 and 4), whereas an extensive distribution of inflammatory cells was observed in group 3 (ligated periosteal flap). The formation of multinucleated giant cells in contact to the $\beta$-TCP-scaffold was partly observed in group 2 (flap $+\beta$-TCP) and group 4 (flap $+\beta$-TCP scaffold + MSCs/EPCs) (Fig. 6 and Table I). 

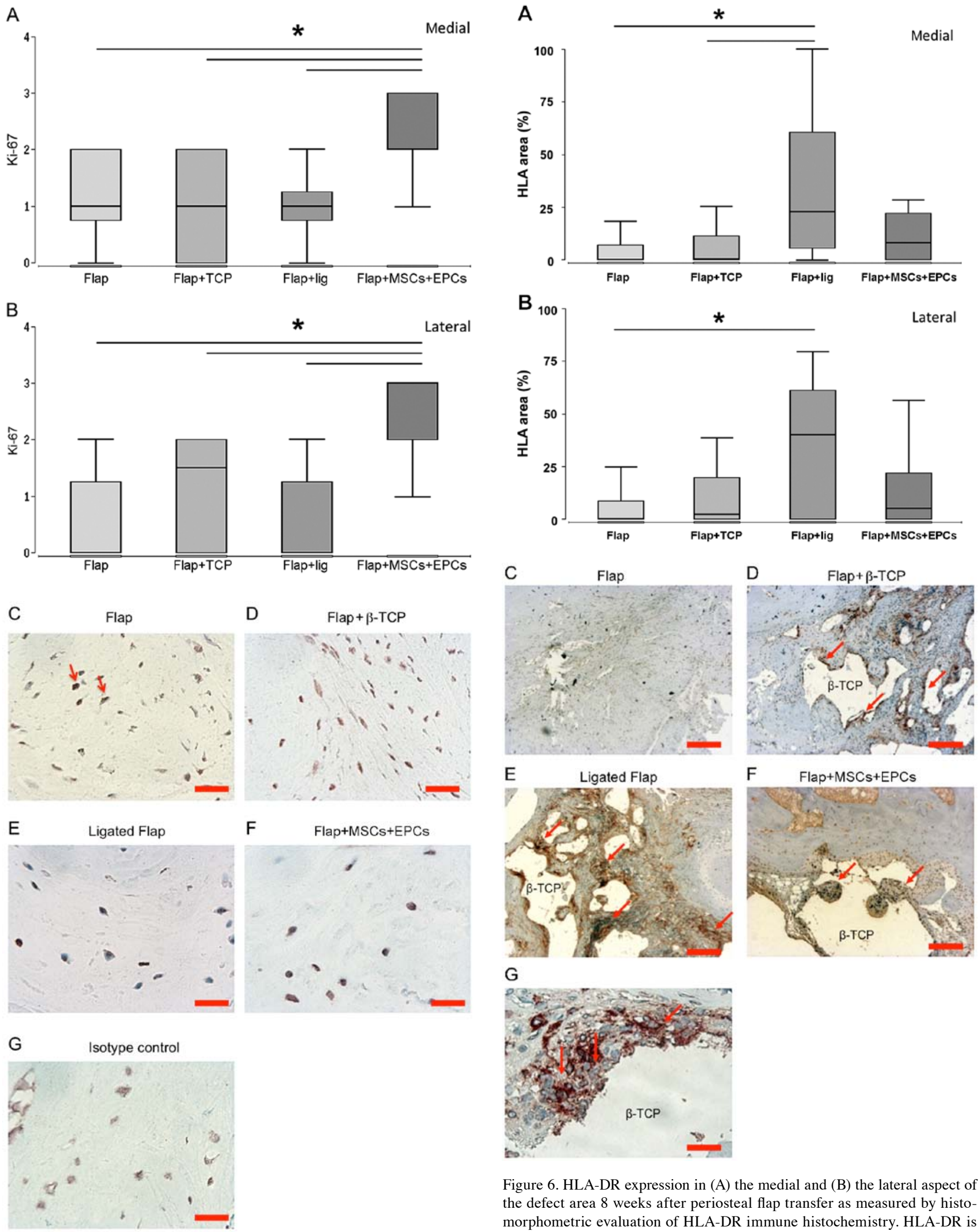

Figure 6. HLA-DR expression in (A) the medial and (B) the lateral aspect of the defect area 8 weeks after periosteal flap transfer as measured by histomorphometric evaluation of HLA-DR immune histochemistry. HLA-DR is expressed on activated immune cells and indicates inflammatory processes. Significantly increased HLA-DR expression was observed, if the perios-

Figure 5. Proliferative activity in (A) the medial and (B) the lateral aspect of the defect zone as determined by Ki-67 immune staining. Histological score values are presented (1, weak proliferative activity; 4 , high proliferative activity). (C-G) Representative histological slides are shown. Ki-67-positive nuclei were identified by a more blackened staining [as exemplarily marked by blue arrows in $(\mathrm{C})$ ]. (G) Isotype control is shown. Red bar represents $25 \mu \mathrm{m}$. teal flap was ligated. (C-F) Representative histological slides are shown. HLA-DR-positive cells accumulate predominantly in direct vicinity to the $\beta$-TCP granules. (F) Formation of multinucleated giant in contact zone to the $\beta$-TCP granules is exemplarily shown. Red bar represents (C-F) $200 \mu \mathrm{m}$, (G) $25 \mu \mathrm{m}$. Arrows indicate accumulations of (C-F) HLA-DR-positive cells and $(\mathrm{G})$ giant cells. ${ }^{*} \mathrm{P}<0.05$. 
Table I. P-value overview.

\begin{tabular}{|c|c|c|c|c|c|c|c|}
\hline Group comparison & BMD & Force & CD31 & Ki-67 med. & Ki-67 lat. & HLA med. & HLA lat. \\
\hline Flap vs. flap $+\beta$-TCP & 0.31 & 0.53 & 0.92 & 0.64 & 0.23 & 0.56 & 0.53 \\
\hline Flap vs. flap + lig & 0.02 & 0.8 & 0.44 & 0.52 & 0.81 & 0.001 & 0.01 \\
\hline Flap vs. flap + MSCs + EPCs & 0.56 & 0.14 & 0.12 & 0.003 & 0.0003 & 0.16 & 0.27 \\
\hline Flap + $\beta$-TCP vs. flap + lig & 0.22 & 0.38 & 0.62 & 0.89 & 0.37 & 0.008 & 0.056 \\
\hline Flap $+\beta$-TCP vs. flap + MSCs + EPCs & 0.11 & 0.44 & 0.21 & 0.003 & 0.016 & 0.39 & 0.59 \\
\hline Flap + lig vs. flap + MSCs + EPCs & 0.005 & 0.007 & 0.036 & 0.001 & 0.0003 & 0.13 & 0.24 \\
\hline
\end{tabular}

Significance is determined as $\mathrm{P}<0.05$. BMD, bone mineral density; force, biomechanical stability force; lat., laterial; med., medial; MSCs, mesenchymal stem cells; EPCs, endothelial progenitor cells. CD31, Ki-67 and HLA indicate staining with the respective antibodies.

\section{Discussion}

Autologous bone grafts continue to be the 'gold standard' for the treatment of large bone defects. Their effectiveness has been attributed to the bone- and vessel-forming cells and growth factors they contain and furthermore to their 3D structure which helps bridge the gap left due to missing bone tissue. Most new treatments developed to substitute autologous bone grafts have been designed to emulate these qualities with the goal of matching or exceeding their effectiveness. With this goal in mind, in the present study, we tested a combination of bone and vessel forming cells, a 3D scaffold and a vascularized periosteal tissue flap in a rat femur critical size defect model.

In the present experiments, our vascularized periosteal flap provided periosteal tissue itself, with its recognized role in bone growth and repair, a rich blood supply and its 3D scaffold-like structure that bridged the gap between the proximal and distal femur bone/periosteum on either side of the defect. The periosteum plays an important role in bone healing. In larger bone defects the periosteum of the distal and proximal edges of the defect grow towards one another in an attempt to bridge the gap resulting from missing bone. However, when the defect is too large and the distance between the distal and proximal defect edges is too great, the ability of the periosteum to bridge the gap is overpowered and healing is delayed or does not occur at all. In these cases, by providing a connection between the periosteum at the distal and proximal edges of the defect, a periosteal flap can help to span the gap and in doing so promote healing. Periosteum can be transplanted as a whole tissue on a vascular pedicle, when placed in a large bone defect, and provides the following properties: its bone growth/repair capabilities, a rich blood supply, and its 3D structure that serves as a scaffold upon which locally generated, systemically derived or transplanted stem cells and/or growth factors can adhere. Taking advantage of all these properties, vascularized periosteal flaps have been used successfully to treat difficult cases of persistent pseudarthrosis, radiation and avascular necrosis and even large bony defects created by debridement of osteomyelitis, pseudarthrosis and infections $(12,42,43)$.

In the present study all animals treated with vascularized periosteal flaps exhibited a significant increase in bone formation in the defect, as measured quantitatively by an increase in BMD and evaluated qualitatively by osteocalcin immunostaining when compared to group 3 in which the vascular pedicle was ligated. This effect was greatest in the defects treated with MSCs, EPCs and $\beta$-TCP scaffold, and to a lesser degree in those that received scaffold solely. As a limitation to mention, no additional staining was performed to provide more information regarding tissue composition. This would be an interesting approach for further studies.

These findings coincide with those of Vögelin et al, who found in a similar rat femur critical-sized defect model that the combination of OPLA-HY scaffold, rhBMP-2 and a vascularized periosteal flap resulted in new callus formation and bony bridging (12).

In another study, Camilli and Penteado harvested a periosteal flap from the medial femoral condyle, created a pouch with it and filled it with cortical bone in one group. One out of each group was connected to the femoral bone and the other part was buried in soft tissue. They described that the presence of a periosteal flap, adjacent to living cortical bone, favours bone formation in contrast to the ones buried in soft tissue. This effect was even greater if the pouch was additionally filled with cortical bone (44).

In the present study, to differentiate between the role played by the blood supply of the flap vs. the periosteal tissue itself, in one group, after lifting and transferring the flap into the bone defect, we ligated its vascular pedicle. Not surprisingly, we found that instead of newly formed bone, the defect contained necrotic fibrous tissue. In addition, in this group of animals, we observed an accumulation of activated HLA-DR-expressing cells and plate loosening. These observations highlight the important role which the flap blood supply plays in bone healing and the prevention of overwhelming inflammation that is probably caused by the necrotic tissue.

A margin of inflammatory cells was observed around the $\beta$-TCP granules in groups 2 and 4 . The accumulation of inflammatory cells, and the generation of multinucleated giant cells should be rated as a foreign body response as it is being triggered by various $\beta$-TCP scaffolds as described by Ghanaati et al (45).

In the present study, we used $\beta$-TCP scaffold to fill the physical gap created by missing bone in our rat femur critical-sized defect model. While their constitution is not comparable to the osteoconductive and osteoinductive nature of autologous bone, bone graft substitutes do eliminate donor-site morbidity and material limitations $(5,46)$. We chose this particular scaffold 
as in previous in vitro studies, we compared several different commercially available bone graft substitutes in combination with MSCs + EPCs and found $\beta$-TCP to be superior compared to other synthetic biomaterials in its osteoconductive and osteoinductive properties (number of invading cells, cell location, differentiation rate and potential) $(3,20,47)$.

In previous experiments, using the same rat model, we demonstrated that MSCs promote bone healing. Moreover, by adding EPCs and MSCs, we demonstrated a further improvement in bone healing. In mechanically unstable breaks, the lack of vasculature causes the bulk of the MSCs to develop into bridging cartilage that eventually spans the defect and then is further stabilized by a surrounding bony bridge. It is therefore necessary not only to provide sufficient number of MSCs in the bone defect zone, but also vascularity and EPCs to promote direct callus formation. We showed that MSCs alone caused an increase of new bone mass, whereas adding EPCs resulted in both increased bone mass and improved angiogenesis probably mediated by the release of VEGF through EPCs (17-19).

Additionally, it has been reported that human MSCs secrete a distinct set of cytokines constitutively, such as VEGF, interleukin (IL)-6 or transforming growth factor (TGF)- $\beta$, which stimulate reparative events and inhibit degenerative events (48-50). Thus, conceivably, MSCs could exert therapeutic effects by this cytokine secretory activity alone. For example, it has been described that 'mesenchymal stem cells support migration, extracellular matrix invasion, proliferation, and survival of endothelial cells in vitro' by the secretion of VEGF, basic fibroblast growth factor (bFGF), angiogenin, procathepsin B, IL-11, and BMP-2 (51).

The combined secretion of various growth factors through MSCs and EPCs might be the reason for the elevated proliferative activity as an indicator for an increased regenerative response observed in group 4 (flap $+\beta-\mathrm{TCP}+\mathrm{MSCs} / \mathrm{EPCs}$ ) of the present study.

Hence, in comparison to other studies on bone healing where only BMP was added to a periosteal flap (12), the bone healing response in the present study benefits additionally from the cytokine secretory capabilities of MSCs and EPCs.

In this study, we found similar results for bone healing in the groups with the flap alone and when MSCs/EPCs are added. We can therefore assume that the vascularized flap already seems to bring the most important characteristics with it. This emphasizes the role of the periosteum which includes a wide range of stem cells as mentioned earlier and the vascularization.

Proliferating cells represented by Ki-67 staining showed significantly highest amounts in group 4 (flap $+\beta$-TCP + MSCs/EPCs) in comparison to all the other groups. Since BMD and biomechanical stability were highest in this group we assume a direct correlation to these proliferating cells. It is not absolutely clear what type of cells are represented by Ki-67 staining. They are probably the highly active MSCs themselves, but also osteoblasts since they can also be found in the callus directly (52).

In conclusion, we combined MSCs, EPCs, $\beta$-TCP scaffold and a vascularized periosteal flap in the present study to improve osteoconductive and osteoinductive qualities inherently present in autologous bone grafts.

\section{Acknowledgements}

This project was funded in part by a LOEWE Center for Cell and Gene Therapy Frankfurt grant from the Hessian Ministry of Higher Education, Research, and the Arts [ref no. III L 4-518/17.004 (2010)] and the Friedrichsheim Foundation in Frankfurt, Germany.

\section{References}

1. Bauer TW and Muschler GF: Bone graft materials. An overview of the basic science. Clin Orthop Relat Res 371: 10-27, 2000.

2. Horner EA, Kirkham J, Wood D, Curran S, Smith M, Thomson B and Yang XB: Long bone defect models for tissue engineering applications: Criteria for choice. Tissue Eng Part B Rev 16: 263-271, 2010.

3. Giannoudis PV, Dinopoulos H and Tsiridis E: Bone substitutes: An update. Injury 36 (Suppl 3): S20-S27, 2005.

4. Nandi SK, Roy S, Mukherjee P, Kundu B, De DK and Basu D: Orthopaedic applications of bone graft \& graft substitutes: A review. Indian J Med Res 132: 15-30, 2010.

5. Finkemeier CG: Bone-grafting and bone-graft substitutes. J Bone Joint Surg Am 84-A: 454-464, 2002.

6. Iacobellis C, Berizzi A and Aldegheri R: Bone transport using the Ilizarov method: A review of complications in 100 consecutive cases. Strateg Trauma Limb Reconstr 5: 17-22, 2010.

7. Doi K and Sakai K: Vascularized periosteal bone graft from the supracondylar region of the femur. Microsurgery 15: 305-315, 1994.

8. SoldadoF, Garcia Fontecha C, Haddad S, Hernandez-Fernandez A, Corona $\mathrm{P}$ and Guerra-Farfan E: Treatment of congenital pseudarthrosis of the tibia with vascularized fibular periosteal transplant. Microsurgery 32: 397-400, 2012.

9. Matsumura G, Hibino N, Ikada Y, Kurosawa H and Shin'oka T: Successful application of tissue engineered vascular autografts: Clinical experience. Biomaterials 24: 2303-2308, 2003.

10. Doi M, Nagano A and Nakamura Y: Genome-wide screening by cDNA microarray of genes associated with matrix mineralization by human mesenchymal stem cells in vitro. Biochem Biophys Res Commun 290: 381-390, 2002.

11. Marcacci M, Kon E, Moukhachev V, Lavroukov A, Kutepov S, Quarto R, Mastrogiacomo M and Cancedda R: Stem cells associated with macroporous bioceramics for long bone repair: 6- to 7-year outcome of a pilot clinical study. Tissue Eng 13: 947-955, 2007.

12. Vögelin E, Jones NF, Huang JI, Brekke JH and Lieberman JR: Healing of a critical-sized defect in the rat femur with use of a vascularized periosteal flap, a biodegradable matrix, and bone morphogenetic protein. J Bone Joint Surg Am 87: 1323-1331, 2005.

13. Mastrogiacomo M, Scaglione S, Martinetti R, Dolcini L, Beltrame F, Cancedda R and Quarto R: Role of scaffold internal structure on in vivo bone formation in macroporous calcium phosphate bioceramics. Biomaterials 27: 3230-3237, 2006.

14. Nauth A, Giannoudis PV, Einhorn TA, Hankenson KD, Friedlaender GE, Li R and Schemitsch EH: Growth factors: Beyond bone morphogenetic proteins. J Orthop Trauma 24: 543-546, 2010.

15. Tang Y, Tang W, Lin Y, Long J, Wang H, Liu L and Tian W: Combination of bone tissue engineering and BMP-2 gene transfection promotes bone healing in osteoporotic rats. Cell Biol Int 32: 1150-1157, 2008.

16. Madeddu P: Therapeutic angiogenesis and vasculogenesis for tissue regeneration. Exp Physiol 90: 315-326, 2005.

17. Henrich D, Seebach C, Kaehling C, Scherzed A, Wilhelm K, Tewksbury R, Powerski M and Marzi I: Simultaneous cultivation of human endothelial-like differentiated precursor cells and human marrow stromal cells on beta-tricalcium phosphate. Tissue Eng Part C Methods 15: 551-560, 2009.

18. Seebach C, Henrich D, Kähling C, Wilhelm K, Tami AE, Alini M and Marzi I: Endothelial progenitor cells and mesenchymal stem cells seeded onto beta-TCP granules enhance early vascularization and bone healing in a critical-sized bone defect in rats. Tissue Eng Part A 16: 1961-1970, 2010.

19. Seebach C, Henrich D, Wilhelm K, Barker JH and Marzi I: Endothelial progenitor cells improve directly and indirectly early vascularization of mesenchymal stem cell-driven bone regeneration in a critical bone defect in rats. Cell Transplant 21: $1667-1677,2012$. 
20. Seebach C, Schultheiss J, Wilhelm K, Frank J and Henrich D: Comparison of six bone-graft substitutes regarding to cell seeding efficiency, metabolism and growth behaviour of human mesenchymal stem cells (MSC) in vitro. Injury 41: 731-738, 2010.

21. Gamradt SC and Lieberman JR: Genetic modification of stem cells to enhance bone repair. Ann Biomed Eng 32: 136-147, 2004

22. Keramaris NC, Calori GM, Nikolaou VS, Schemitsch EH and Giannoudis PV: Fracture vascularity and bone healing: A systematic review of the role of VEGF. Injury 39 (Suppl 2): S45-S57, 2008.

23. Guo X, Zheng Q, Kulbatski I, Yuan Q, Yang S, Shao Z, Wang H, $X$ iao $B$, Pan $Z$ and Tang S: Bone regeneration with active angiogenesis by basic fibroblast growth factor gene transfected mesenchymal stem cells seeded on porous beta-TCP ceramic scaffolds. Biomed Mater 1: 93-99, 2006.

24. Peng H, Wright V, Usas A, Gearhart B, Shen HC, Cummins J and Huard J: Synergistic enhancement of bone formation and healing by stem cell-expressed VEGF and bone morphogenetic protein-4. J Clin Invest 110: 751-759, 2002.

25. Kumar S, Wan C, Ramaswamy G,Clemens TL and Ponnazhagan S: Mesenchymal stem cells expressing osteogenic and angiogenic factors synergistically enhance bone formation in a mouse model of segmental bone defect. Mol Ther 18: 1026-1034, 2010.

26. Asahara T, Masuda H, Takahashi T, Kalka C, Pastore C, Silver M, Kearne M, Magner M and Isner JM: Bone marrow origin of endothelial progenitor cells responsible for postnatal vasculogenesis in physiological and pathological neovascularization. Circ Res 85: 221-228, 1999.

27. Atesok K, Li R, Stewart DJ and Schemitsch EH: Endothelial progenitor cells promote fracture healing in a segmental bone defect model. J Orthop Res 28: 1007-1014, 2010.

28. Rozen N, Bick T, Bajayo A, Shamian B, Schrift-Tzadok M, Gabet Y, Yayon A, Bab I, Soudry M and Lewinson D: Transplanted blood-derived endothelial progenitor cells (EPC) enhance bridging of sheep tibia critical size defects. Bone 45: 918-924, 2009.

29. Jones AL, Bucholz RW, Bosse MJ, Mirza SK, Lyon TR, Webb LX, Pollak AN, Golden JD and Valentin-Opran A; BMP-2 Evaluation in Surgery for Tibial Trauma-Allgraft (BESTT-ALL) Study Group: Recombinant human BMP-2 and allograft compared with autogenous bone graft for reconstruction of diaphyseal tibial fractures with cortical defects. A randomized, controlled trial. J Bone Joint Surg Am 88: 1431-1441, 2006.

30. Daniels T, DiGiovanni C, Lau JT, Wing K and Younger A: Prospective clinical pilot trial in a single cohort group of rhPDGF in foot arthrodeses. Foot Ankle Int 31: 473-479, 2010

31. O'Driscoll SW and Fitzsimmons JS: The role of periosteum in cartilage repair. Clin Orthop Relat Res (Suppl): S190-S207, 2001.

32. O'Driscoll SW, Saris DB, Ito Y and Fitzimmons JS: The chondrogenic potential of periosteum decreases with age. J Orthop Res 19: 95-103, 2001.

33. Jaffe HL (ed): Metabolic, Degenerative and Inflammatory Diseases of Bone and Joints. 1st edition. Urban and Schwarzenberg, München-Berlin-Wien, 1972.

34. Dwek JR: The periosteum: What is it, where is it, and what mimics it in its absence? Skeletal Radiol 39: 319-323, 2010

35. Allen MR, Hock JM and Burr DB: Periosteum: Biology, regulation, and response to osteoporosis therapies. Bone 35 1003-1012, 2004.

36. Nau C, Henrich D, Seebach C, Schröder K, Fitzsimmons SJ, Hankel S, Barker JH, Marzi I and Frank J: Treatment of large bone defects with a vascularized periosteal flap in combination with biodegradable scaffold seeded with bone marrow-derived mononuclear cells: An experimental study in rats. Tissue Eng Part A 22: 133-141, 2016.
37. Arthur A, Zannettino A and Gronthos S: The therapeutic applications of multipotential mesenchymal/stromal stem cells in skeletal tissue repair. J Cell Physiol 218: 237-245, 2009.

38. Eldesoqi K, Henrich D, El-Kady AM, Arbid MS, AbdEl-Hady BM, Marzi I and Seebach C: Safety evaluation of a bioglass-polylactic acid composite scaffold seeded with progenitor cells in a rat skull critical-size bone defect. PLoS One 9: e87642, 2014.

39. Eldesoqi K, Seebach C, Nguyen Ngoc C, Meier S, Nau C, Schaible A, Marzi I and Henrich D: High calcium bioglass enhances differentiation and survival of endothelial progenitor cells, inducing early vascularization in critical size bone defects PLoS One 8: e79058, 2013.

40. Henrich D, Hahn P, Wahl M, Wilhelm K, Dernbach E, Dimmeler S and Marzi I: Serum derived from multiple trauma patients promotes the differentiation of endothelial progenitor cells in vitro: Possible role of transforming growth factor-betal and vascular endothelial growth factor165. Shock 21: 13-16, 2004.

41. Henrich D, Seebach C, Wilhelm K and Marzi I: High dosage of simvastatin reduces TNF-alpha-induced apoptosis of endothelial progenitor cells but fails to prevent apoptosis induced by IL-1beta in vitro. J Surg Res 142: 13-19, 2007.

42. Del Piñal F, García-Bernal FJ, Regalado J, Ayala H, Cagigal L and Studer A: Vascularised corticoperiosteal grafts from the medial femoral condyle for difficult non-unions of the upper limb. J Hand Surg Eur Vol 32: 135-142, 2007.

43. Fuchs B, Steinmann SP and Bishop AT: Free vascularized corticoperiosteal bone graft for the treatment of persistent nonunion of the clavicle. J Shoulder Elbow Surg 14: 264-268, 2005.

44. Camilli JA and Penteado CV: Bone formation by vascularized periosteal and osteoperiosteal grafts. An experimental study in rats. Arch Orthop Trauma Surg 114: 18-24, 1994.

45. Ghanaati S, Barbeck M, Orth C, Willershausen I, Thimm BW, Hoffmann C, Rasic A, Sader RA, Unger RE and Peters F: Influence of $\beta$-tricalcium phosphate granule size and morphology on tissue reaction in vivo. Acta Biomater 6: 4476-4487, 2010

46. Faour O, Dimitriou R, Cousins CA and Giannoudis PV: The use of bone graft substitutes in large cancellous voids: Any specific needs? Injury 42 (Suppl 2): S87-S90, 2011.

47. Schultheiss J, Seebach C, Henrich D, Wilhelm K, Barker JH and Frank J: Mesenchymal stem cell (MSC) and endothelial progenitor cell (EPC) growth and adhesion in six different bone graft substitutes. Eur J Trauma Emerg Surg 37: 635-644, 2011.

48. Lee DE, Ayoub N and Agrawal DK: Mesenchymal stem cells and cutaneous wound healing: Novel methods to increase cell delivery and therapeutic efficacy. Stem Cell Res Ther 7: 37, 2016.

49. Zhao L, Liu X, Zhang Y, Liang X, Ding Y, Xu Y, Fang Z and Zhang F: Enhanced cell survival and paracrine effects of mesenchymal stem cells overexpressing hepatocyte growth factor promote cardioprotection in myocardial infarction. Exp Cell Res 344: 30-39, 2016

50. Merino-González C, Zuñiga FA, Escudero C, Ormazabal V, Reyes C, Nova-Lamperti E, Salomón C and Aguayo C: Mesenchymal stem cell-derived extracellular vesicles promote angiogenesis: Potencial clinical application. Front Physiol 7: 24, 2016.

51. Potapova IA, Gaudette GR, Brink PR, Robinson RB, Rosen MR, Cohen IS and Doronin SV: Mesenchymal stem cells support migration, extracellular matrix invasion, proliferation, and survival of endothelial cells in vitro. Stem Cells 25: 1761-1768, 2007.

52. Hoch AI, Binder BY, Genetos DC and Leach JK: Differentiation-dependent secretion of proangiogenic factors by mesenchymal stem cells. PLoS One 7: e35579, 2012. 